\title{
Ultrasmall particle detection using a submicron Hall sensor
}

O. Kazakova,1,a_V. Panchal,1,2J. Gallop,1 P. See,1,3 D. C. Cox,1,4 M. Spasova,5 and

L. F. Cohen6

1National Physical Laboratory, Teddington TW11 0LW, United Kingdom

2Royal Holloway, University of London, Egham TW20 0EX, United Kingdom

3University of Cambridge, Cambridge CB3 OHE, United Kingdom

4University of Surrey, Guildford GU2 7XH, United Kingdom

5Center for Nanointegration, University of Duisburg-Essen, Duisburg 47048, Germany

6Imperial College London, London SW7 2BW, United Kingdom

We demonstrate detection of a single FePt nanoparticle _diameter $150 \mathrm{~nm}$, moment $\sim 107$ $\mu$ в using an ultrasensitive InSb Hall sensor with the bar lateral width of $600 \mathrm{~nm}$. The white noise of a typical nanodevice, Sv 1/2_28 nV/_ _Hz, is limited only by two-terminal resistance of the voltage leads which results in a minimum field sensitivity of the device Bmin $=0.87 \_\mathrm{T} /{ }_{-} \mathrm{Hz}$. To detect a single FePt bead, we employed a phase-sensitive method based on measuring the ac susceptibility change in a bead when exposed to a switched dc magnetic field. Such nano-Hall devices, enabling detection of potentially even smaller moments, are of considerable significance both for nanomagnetic metrology and high sensitivity biological and environmental detectors. 
Properties of magnetic nanoparticles have been a subject of intensive theoretical and experimental studies since the late 1940s. Recently, various magnetic properties of individual particles and nanowires were successfully studied using a number of techniques, including GMR sensors, dc nano-SQUIDs _superconducting quantum interference device, and Hall magnetometry. All the methods listed above have their own advantages and vary in terms of magnetic sensitivity, applicability to particular magnetic systems/ experimental conditions and complexity. Small Hall sensors operating at room temperature are preferred for numerous biomedical applications. The main advantages of semiconductor Hall sensors are their noninvasive nature and high magnetic field sensitivity.1,2 Such sensors are already an essential part of many existing and prospective detection schemes for the biomedical industry, allowing a straightforward method for basic research into molecular recognition processes with potential single molecule resolution.

Among novel applications, it was proposed that a small submicronsized Hall sensor could be an integrated part of a generic biosensor/nanoactuator device.3 Depending on the specific application, magnetic particles used as biological labels can be prepared in a variety of sizes and have an advantage of controllable tagging. For a number of applications_both related to biomedical and information technology_IT_industries a precise knowledge of magnetic properties on the nanoscale is required. Despite enormous progress in the understanding of magnetic phenomena achieved so far, many fundamental questions are still unanswered. Among them are metrological issues related to measurements of ultrasmall magnetic moments. Thus, the magnetic investigation of a single nanoparticle is of special interest both from a fundamental/metrological point of view and for existing and potential applications, including magnetic recording, biomedical applications, and environmental monitoring.

Here, we present our results on detection of a small FePt nanoparticle with diameter of $150 \mathrm{~nm}$ and a magnetic moment of about 107 _ $\mathrm{B}$ using an ultrasensitive and noninvasive InSb Hall sensor. Although a number of attempts have been undertaken to detect small paramagnetic particles4-6 and even studies of the magnetization reversal in small ferromagnetic particles were reported previously, 7 we present the first submicron-size Hall sensor capable of detecting an individual magnetic nanobead_composed of 
_5_1044 nm size nanoparticles_ at room temperature. Undoped InSb films about 300 $\mathrm{nm}$ thick were grown by two-phase molecular-beam epitaxy on semi-insulating GaAs _001_ substrates at a base pressure of 10-10 mbar. The growth procedure was adopted from Ref. 8. Initially a lowtemperature _ ${ }_{-}{ }_{g r}=300{ }^{\circ} \mathrm{C}_{-}$InSb passive buffer layer _approximately $20 \mathrm{~nm}_{-}$was deposited followed by the growth of an undoped InSb active layer_ $\mathrm{T}_{\mathrm{gr}}=380^{\circ} \mathrm{C}_{-}$. The method allows a significant improvement of structural and electronic properties of the InSb films. A high carrier_electron_mobility, $\quad=1.3 \mathrm{~m} 2$ $/ \mathrm{V}$ s, and concentration, n_3.9_1016 cm-3, were deduced from magnetoresistance measurements in a perpendicular magnetic field in the van der Pauw geometry. Double Hall crosses with bar widths in the range of $600 \mathrm{~nm}-5$ _m were fabricated by e-beam lithography and reactive ion etching_Fig. 1, left_. The ohmic contacts were formed using a nonannealed titanium/gold layer on the electrical pads. As a result, a 600-nm sized Hall device has fourterminal and two-terminal resistances of $6.5 \mathrm{kOhm}$ and 58 $\mathrm{kOhm}$, respectively. The large two-terminal resistance is a result of long InSb leads. All experiments were performed at room temperature in a dark environment. The device was biased by a battery powered dc current source. As a part of the initial characterization, transverse or Hall _ $\mathrm{V}_{\mathrm{xy}}$ and longitudinal _ $\mathrm{V}_{\mathrm{xx}_{-}}$voltages were measured on all of the devices. The typical value of the Hall coefficient is $\mathrm{RH}_{2} 660 \mathrm{Ohm} / \mathrm{T}$ _Fig. 2_a_- and magnetoresistance is MR $\quad=4.7 \%$ for the as prepared devices with a smallest size of $600 \mathrm{~nm}$. All adjacent Hall crosses demonstrate very similar characteristics with the difference in the Hall coefficient _ $\mathrm{RH}_{-} 5 \%$ _where the smallest devices demonstrate the largest value $_{-}$, making these devices suitable for simultaneous measurements, when the magnetic particle is placed on cross no. 1 , while cross no. 2 is left empty as a control experiment.

The Hall coefficient is weakly dependent on the size of the device, being RH_660 and 850 $\mathrm{Ohm} / \mathrm{T}$ for crosses with bar widths of $600 \mathrm{~nm}$ and 5 _m, respectively. This decrease in Hall coefficient is associated with the stronger influence of interfaces and increased scattering of carriers in smaller devices, which lead to changes in mobility in the interfacial regions.

For noise measurements, the transverse voltage leads were directly connected to the input of a low frequency fast Fourier transform spectrum analyzer. All Hall devices 
exhibit a $1 /$ flike noise spectrum where the position of the 1 / f-noise corner is proportional to the bias current and inversely proportional to the size of the Hall sensor _Fig. 2_b_-. Above this threshold, the noise is independent of frequency, bias current, and dc magnetic field, i.e., it is essentially white noise. For a typical $600-\mathrm{nm}$ wide device the white noise is in order of $\mathrm{Sv} 1 / 2 \_28 \mathrm{nV} /{ }_{-} \mathrm{Hz}$ at $\mathrm{f}_{-} 70 \mathrm{~Hz}$ and zero bias current_Fig. $2 \_b_{--}$and limited only by the relatively high two-terminal lead resistance of the Hall sensor. Thus, the minimum field sensitivity of the device achievable at high frequencies or low bias currents _white noise level_ is defined as $B_{\min }=0.87 \_\mathrm{T} /{ }_{-} \mathrm{Hz}$. However, at lower frequencies_ $1 /$ f noise _ the spectral noise rapidly increases with the bias current and is inversely proportional to the bar width_Figs. 2_b_ and 2_c_-.

FePt beads were fabricated from the water-based solution of FePt nanoparticles. In the first step, FePt nanoparticles were synthesized in hexane by a high-temperature organometallic route using oleic acid and oleic amine as stabilizers.9 Then the particles were transferred into water using tetramethylammonium hydroxide as a phase transfer agent.10 The water-based nanoparticle dispersion was placed into an ultrasound bath at $80^{\circ} \mathrm{C}$ for $1.5 \mathrm{~h}$ that led to formation of beads_ $70-200 \mathrm{~nm}$ in diameter_composed of the FePt nanoparticles as shown in Fig. 1 inset. TEM analysis shows that the beads consist of a large number of closed packed FePt nanoparticles_in an fcc chemically disordered state $_{-}$, with diameters of $3-4 \mathrm{~nm}$ and side-by-side distances of $\_0.5 \mathrm{~nm}$. The energy dispersive $\mathrm{x}$-ray spectroscopy reveals the 40:60 composition of a nanoparticle _Fe $40 \mathrm{Pt} 60 \_$.

Magnetization measurements were performed on a large ensemble of FePt beads and demonstrated their superparamagnetic properties at room temperature. The magnetic moment of the $150 \mathrm{~nm}$ sphere is $\_6 \_107 \_$B in a field of $\mathrm{H} \quad=30 \mathrm{kOe}$ as estimated by SQUID magnetometry and x-ray magnetic circular dichroism _XMCD_measurements.11 A single FePt bead with a size of $150 \mathrm{~nm}$ was positioned on the top of one the Hall sensors using a FEI focused ion beam_FIB_system equipped with a four-probe Zyvex nanomanipulation system. The second cross was left empty as a control experiment. A Si membrane fabricated in the FIB with a hole in its center was used to keep the bead in place and protect the $\mathrm{InSb}$ sensor from elevated temperatures and radiation during exposure to the electron beam_Fig. 1 right_. As the membrane is _ $200 \mathrm{~nm}$ thick and the 
hole diameter $\_120 \mathrm{~nm}_{-}$is smaller than the bead, the estimated distance between the bead center and the Hall cross is _225 $\mathrm{nm}$.

For detection of a single FePt bead we have further advanced a phase-sensitive method initially described in Ref. 12. The technique developed here is based on measurements of the nonlinear susceptibility change in a bead exposed to an ac magnetic field _ $B_{a c}=1.1$ $\mathrm{mT}, \mathrm{f}=491 \mathrm{~Hz}_{-}$applied perpendicular to the plane of the Hall sensor, as a function of an external dc magnetic field Bdc, applied parallel to the ac field. The Hall voltage is lock-in detected at the second harmonic _ $2 \mathrm{f}_{-}$of the ac field frequency _Fig. $3_{-}$. Measurements at the second harmonic allow us to avoid the direct inductive pickup commonly occurring at the first harmonic_see e.g., Ref. 1_. While in the original detection scheme _ac and dc fields are orthogonal_12 the amplitude of the $2 \mathrm{f}$ signal is proportional to the $\mathrm{dc}$ magnetization of the bead, in the method developed here _a.c and dc fields are parallel_, the signal at $2 \mathrm{f}$ arises from the nonlinearity of the $\mathrm{M}_{-} \mathrm{B}_{-}$curve and is proportional to the curvature of the M_B_ plot. A model, to be described in more detail elsewhere, has been developed which shows that the second harmonic signal rises from zero at $B_{d c}=0$ to a maximum at a value of $B_{d c}$ corresponding to the maximum curvature of the magnetization curve, before falling toward zero as the magnetization approaches saturation.

The results of an experiment in which $B d c=340 \mathrm{mT}$ was applied in a pulse mode are shown in Fig. 4, where the dc magnetic field was switched on/off three times, each pulse having a duration of $40 \mathrm{~s}$. The cross with the magnetic bead shows a clear step-wise increase in the ac Hall voltage, $V_{H}=150 \mathrm{nV}$, with no detectable signal measured on the empty second cross. Our simulations show the expected step-wise second harmonic signal to be within a factor of 3 of this value, rather reasonable agreement since the signal is very sensitive to both the approach distance between bead center and Hall cross and the active dimensions of the nanoscale cross itself. It should be noted that the operational frequency range of the existing setup is limited to relatively low frequencies.

Limitations of the present detection scheme _i.e., parasitic inductance and capacitance signals at high frequencies_ do not allow operation of the Hall devices at their white noise level _the crossover between $1 / \mathrm{f}$ and white noise occurs at _ $5 \mathrm{kHz}$ for the $600-\mathrm{nm}$ 
device at $\mathrm{IH}_{\mathrm{H}}=50$ _ $\mathrm{A}_{-}$where the sensitivity of the sensor would be maximal. Thus, further improvement of the measurement scheme will allow detection of significantly smaller magnetic moments.

In summary, we have present results on magnetic detection of a single $150 \mathrm{~nm}$ superparamagnetic FePt bead using a submicron-size InSb Hall sensor. Superior performance of the Hall devices studied here opens up new horizons for detection of even smaller magnetic moments, which is of high importance both for metrology of nanomagnetism and for highly sensitive biological and environmental detectors.

\section{ACKNOWLEDGMENTS}

The authors thank A. Trunova _Duisburg-Essen Univ., Germany_ for help in obtaining HRTEM images. This work was supported by the UK DIUS under project ESQ01T1 and by the EC FP6 and FP7 under projects BioNano Switch and NanoSpin.

1G. Mihajlović, P. Xiong, S. von Molnár, K. Ohtani, H. Ohno, M. Field, and G. J. Sullivan, Appl. Phys. Lett. 87, 112502 _2005_.

${ }_{2}$ P. Manandhar, K.-S. Chen, K. Aledealat, G. Mihajlović, C. S. Yun, M. Field, G. J. Sullivan, G. F. Strouse, P. B. Chase, S. von Molnár, and P. Xiong, Nanotechnology 20, 355501 _2009_.

3http://www.nanowerk.com/nanotechnology/labs/BioNano-Switch.html

${ }_{4}$ K. Togawa, H. Sanbonsugi, A. Sandhu, M. Abe, H. Narimatsu, K. Nishio, and H. Handa, Jpn. J. Appl. Phys., Part 2 44, L1494_2005_.

${ }_{5}$ K. Togawa, H. Sanbonsugi, A. Sandhu, M. Abe, H. Narimatsu, K. Nishio, and H. Handa, J. Appl. Phys. 99, $08 \mathrm{P} 103$ _2006_.

${ }_{6}$ M. Mihajlović, P. Xiong, S. von Molnár, M. Field, and G. J. Sullivan, J. Appl. Phys. 102, 034506 _2007_•

7Y. Li, P. Xiong, S. von Molnar, Y. Ohno, and H. Ohno, Phys. Rev. B 71, 214425 _2005_•

8T. Zhang, S. K. Clowes, M. Debnath, A. Bennett, C. Roberts, J. J. Harris, R. A. Stradling, L. F. Cohen, T.

Lyford, and P. F. Fewster, Appl. Phys. Lett. 84, 4463 _2004_.

9S. Sun, C. B. Murray, D. Weller, L. Folks, and A. Moser, Science 287, $1989 \_2000_{-}$.

10V. Salgueiriño-Maceira, L. M. Liz-Marzán, and M. Farle, Langmuir 20, 6946 _2004_.

${ }_{11}$ C. Antoniak, Ph.D. thesis, University Duisburg-Essen, 2007.

${ }_{12}$ P.-A. Besse, G. Boero, M. Demierre, V. Pott, and R. Popovic, Appl. Phys. Lett. 80, 4199_2002_. 


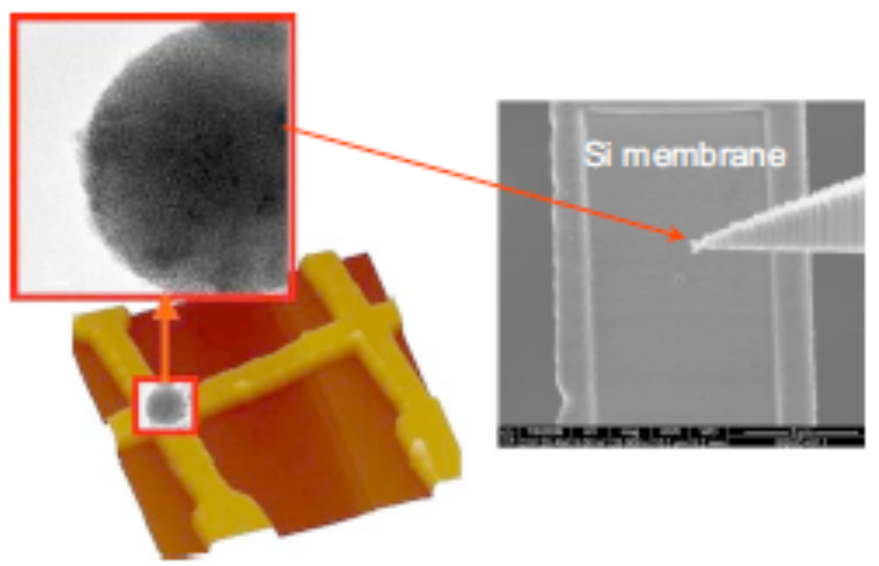

FIG. 1. _Color online_ Left panel: An AFM image of the double-cross InSb Hall sensor with the bar width of $600 \mathrm{~nm}$. A FePt bead _- $150 \mathrm{~nm}_{-}$is highlighted for clarity _not to scale_. Inset: high-resolution transmission electron microscopy image of a bead. Right panel: A Si membrane with a hole in the center was used to keep the bead in place and protect the InSb sensor from elevated temperatures and radiation during the exposure to the electron beam. The FePt particle is located at the end of the manipulation tip just prior to placement in the hole.
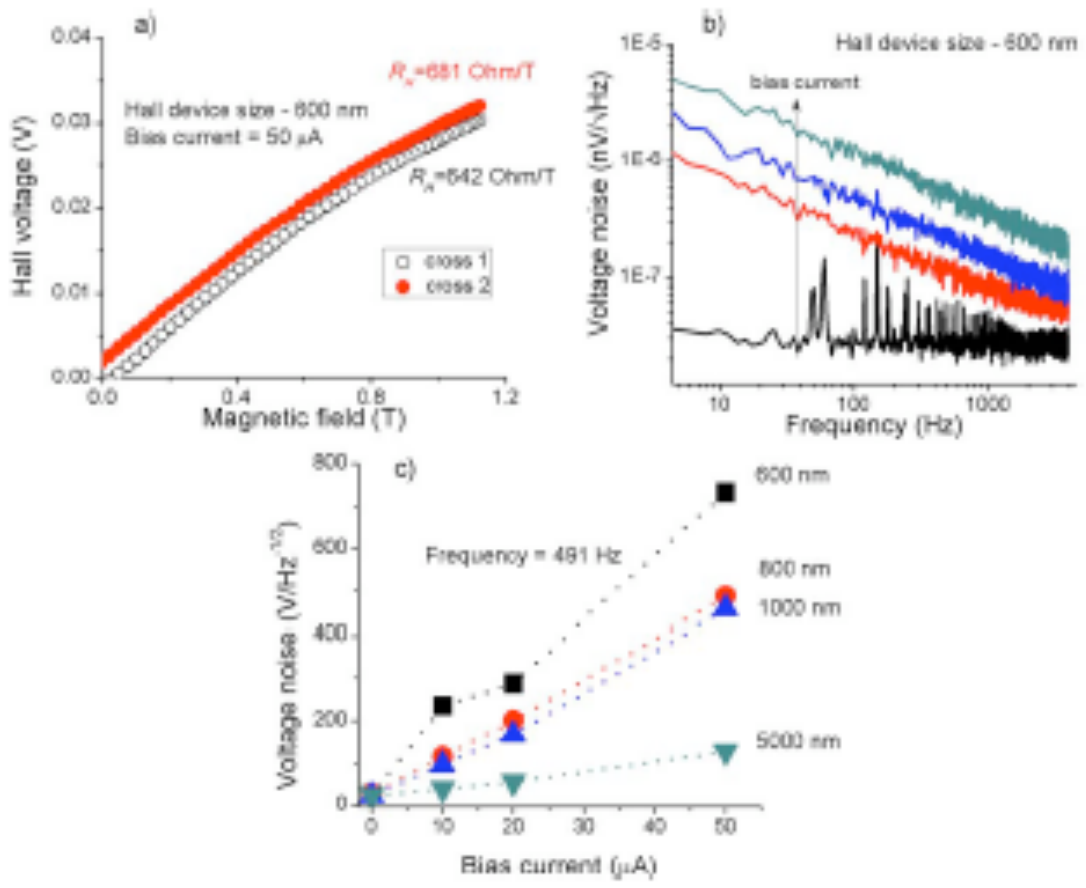

FIG. 2. _a_ Hall coefficient measurements in two adjacent Hall crosses with size $600 \mathrm{~nm}$. _ b_ Spectral noise in the same device, Iн $=0-50 \_$A. _c_ Current dependence of voltage noise in the devices with the lateral size 600 $\mathrm{nm}-5$ _ $\mathrm{m}$ at $\mathrm{f}=491 \mathrm{~Hz}$. 


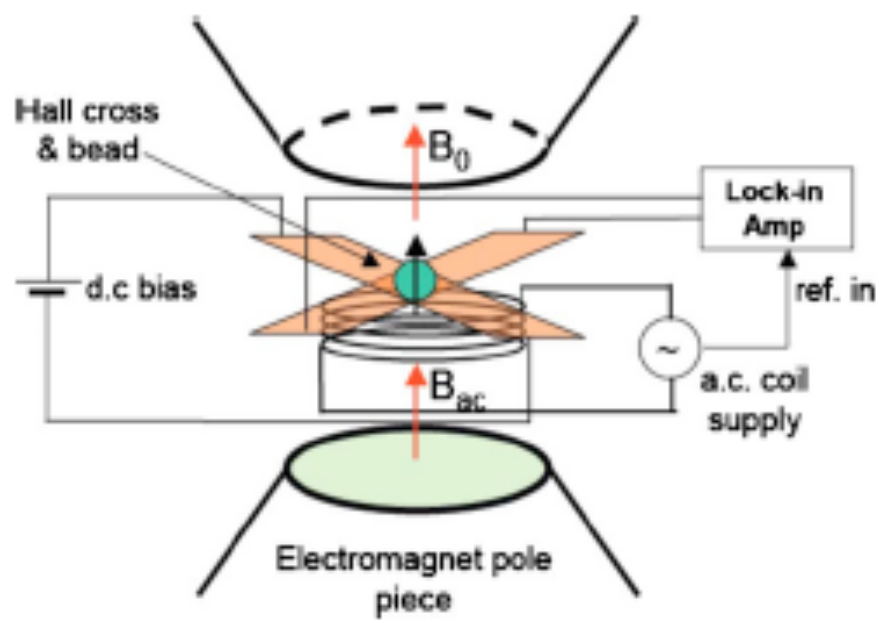

FIG. 3. Schematic of the detection experiment.

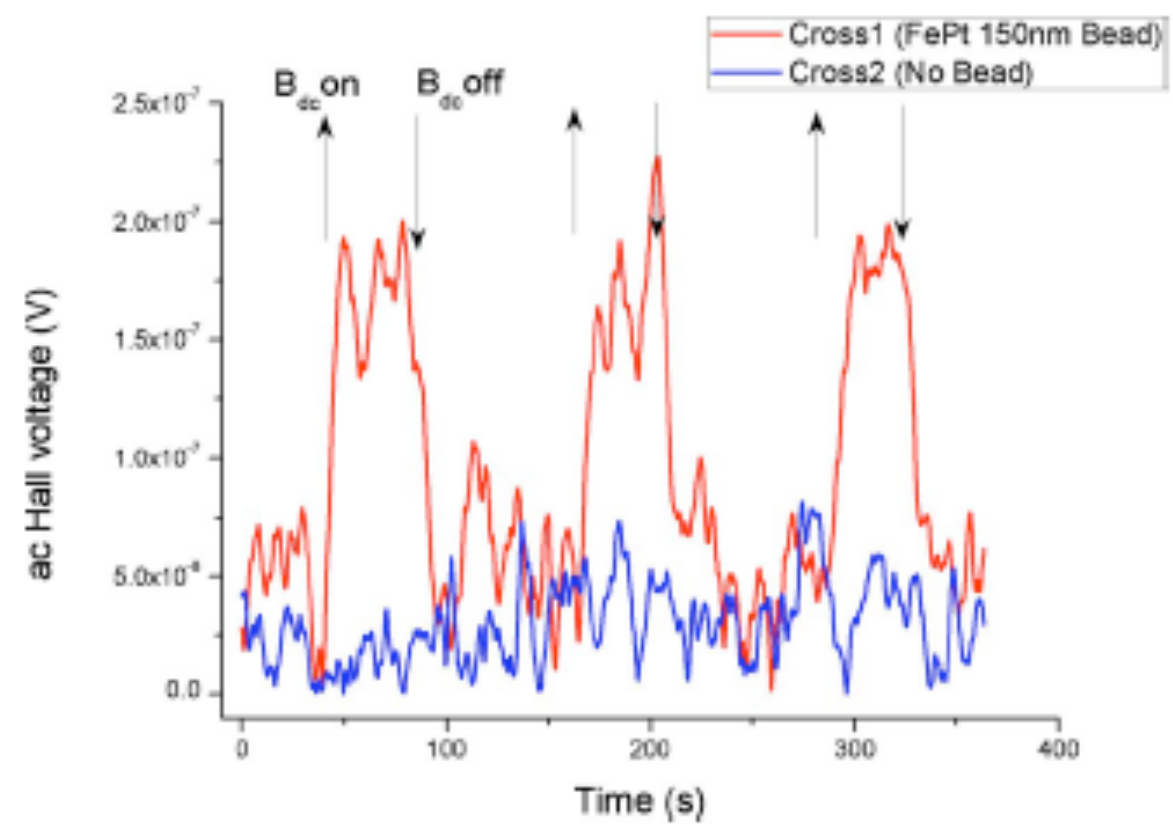

FIG. 4. Detection of an FePt bead, based on the ac-dc measurement technique. Arrows indicate switching of the dc magnetic field on and off, each pulse having a duration of $40 \mathrm{~s}$. The cross with a magnetic bead shows a clear step-wise increase in the ac Hall voltage, $\mathrm{VH}_{-} 150 \mathrm{nV}$. No signal is measured on the empty cross no. 2. 